\title{
Model predictive control based on LPV models with parameter-varying delays
}

\author{
Fatemeh Karimi Pour, Vicenç Puig and Carlos Ocampo-Martinez
}

\begin{abstract}
This paper presents a Model Predictive Control (MPC) strategy based on Linear Parameter Varying (LPV) models with varying delays affecting states and inputs. The proposed control approach allows the controller to accommodate the scheduling parameters and delay change. By computing the prediction of the state variables and delay along a prediction time horizon, the system model can be modified according to the evaluation of the estimated state and delay at each time instant. Moreover, the solution of the optimization problem associated with the MPC design is achieved by solving a series of Quadratic Programming (QP) problem at each time instant. This iterative approach reduces the computational burden compared to the solution of a non-linear optimization problem. A pasteurization plant system is used as a case study to demonstrate the effectiveness of the proposed approach.
\end{abstract}

\section{INTRODUCTION}

In modern times of industrialization, research on advanced control algorithms for complex dynamic systems is still very active. Controlling these complex systems is one of the most important challenges in control engineering. On the other hand, time delays may arise in the dynamics of real systems, such as communication systems, chemical processes, and transportation systems [1]. The effect of time delay in a process increases the control problem. The delays can affect the states, inputs or/and outputs, and they can be timevarying or constant, unknown or known, deterministic or stochastic depending on the considered system. One of the main, successful and popular advanced control strategies for industrial processes is Model Predictive Control (MPC) [2].

MPC based on linear models is typically used in process control where the on-line optimization problem can be formulated as a convex optimization problem by either linear programming or Quadratic Programming (QP). This assumption is suitably considered for typical processes. However, most of the real systems show nonlinear behaviors. In order to reduce the conservativeness, the idea of controlling nonlinear systems considering linear parameter varying (LPV) models has been widely investigated in the literature [3]. The main advantage of LPV models is that the system nonlinearities are embedded into the varying parameters, which make the nonlinear system become a linear-like system with varying parameters [4].

\footnotetext{
*This work has been partially funded by the Spanish State Research Agency (AEI) and the European Regional Development Fund (ERFD) through the projects DEOCS (ref. MINECO DPI2016-76493) and SCAV (ref. MINECO DPI2017-88403-R).

All authors are with Advanced Control Systems Group, Universitat Politècnica de Catalunya, Institut de Robòtica i Informàtica Industrial (CSIC-UPC), C/. Llorens i Artigas 4-6, 08028 Barcelona, Spain ffkarimi, vpuig, cocampo\}eiri.upc.edu
}

Recently, many researchers focuses on the robust model predictive control (RMPC) based on the linear model with constant or varying delays [5], [6]. In [7], a RMPC with constant state delay by using linear matrix inequalities (LMIs) term is proposed. The work of [8] proposes an MPC algorithm for uncertain time varying systems with state delays. Besides, a synthesis strategy for predictive control based on LPV models with state delays was provided in [8]. The results were further extended for the system with input delayed in [9]. However, there exist only a few MPC methods that consider time-delayed LPV model [10], [11]. In [10], authors proposed a parameter-dependent state-feedback controller based on LPV model with parameter-varying time delay and proved the stability by using parameter-dependent Lyapunov functionals. However, there exists a limitation to apply input constraints which are required when controlling many practical plants. Also, an RMPC based on LPV model with state delay was presented in [11] by using a Lyapunov function augmented with the current state and the timedelayed states. But, still delay is considered to be constant in the RMPC.

The main contribution of this paper consists in designing an improved LPV-based MPC strategy in order to formulate an optimization problem that exploits the functional dependency of scheduling variables and varying delays to develop a prediction strategy with the numerically suitable solution. This solution is iteratively forced to an accurate solution, thereby avoiding the use of non-linear optimization. In addition, the optimization problem is decomposed into a series of QP problems that are solved at each time instant. Finally, the small-scale pasteurization plant that presents nonlinear behavior with varying delays is used in order to test the effectiveness of the proposed approach.

The paper is structured as follows. In Section II, the formulation of MPC based on an LPV model with varying delays is introduced. Then, the LPV-based MPC approach for LPV model with varying delay is presented in Section III. In Section IV, results of applying the proposed control strategy to the pasteurization system are presented. Finally, in Section $\mathrm{V}$, the conclusion of this work are drawn.

\section{Problem Statement}

Consider the following discrete-time state-space LPV model with parameter-varying delays in inputs and states:

$$
\begin{aligned}
x(k+1) & =A(\theta(k)) x(k)+A_{\tau}(\theta(k)) x(k-\tau(\theta(k))) \\
& +B(\theta(k)) u(k)+B_{\tau}(\theta(k)) u(k-\tau(\theta(k))), \\
y(k) & =C(\theta(k)) x(k)+C_{\tau}(\theta(k)) x(k-\tau(\theta(k))),
\end{aligned}
$$


where $x \in \mathbb{R}^{n_{x}}, u \in \mathbb{R}^{n_{u}}$ and $y \in \mathbb{R}^{n_{y}}$ are the state vector, input vector and output vector, respectively. Moreover, $A(\theta(k)) \in \mathbb{R}^{n_{x} \times n_{x}}, B(\theta(k)) \in \mathbb{R}^{n_{x} \times n_{u}}$ and $C(\theta(k)) \in \mathbb{R}^{n_{y} \times n_{x}}$ are system matrices with the appropriate dimensions, which depend affinely on the varying parameter $\theta(k) \in \Theta \forall k \geq 0$ where $\Theta$ is a given compact set. Moreover, $\tau$ is a scalar function representing the parameter-varying delay and satisfies $0 \leq \tau_{m} \leq \tau(\theta(k)) \leq \tau_{M}$, where $\tau_{M}$ and $\tau_{m}$ are the upper bound and lower bound of $\tau(\theta(k))$. Throughout this paper, it is assumed that $(A(\theta), B(\theta))$ is stabilizable for all $\theta \in \Theta$.

The MPC controller design with a quadratic objective function subject to input and states constraints based on the LPV model (1) can be formulated as follows:

$$
\min _{\tilde{u}(k)} J(k)=\sum_{i=0}^{N_{p}-1}\|x(i \mid k)\|_{w_{1}}^{p}+\|u(i \mid k)\|_{w_{2}}^{p},
$$

subject to

$$
\begin{aligned}
& x(i+1 \mid k)= A(\theta(i \mid k)) x(i \mid k) \\
&+A_{\tau}(\theta(i \mid k)) x(i-\tau(\theta(i \mid k)) \mid k) \\
&+B(\theta(i \mid k)) u(i \mid k) \\
&+B_{\tau}(\theta(i \mid k)) u(i-\tau(\theta(i \mid k)) \mid k), \\
& u(i \mid k) \in \mathbb{U}, \quad x(i \mid k) \in \mathbb{X}, \\
& x(0 \mid k)= x(k) \quad \theta(0 \mid k)=\theta(i \mid k),
\end{aligned}
$$

and for all $i \in \mathbb{Z}_{\left[0, N_{p}-1\right]}$, it is solved online for $\tilde{u}(k)=$ $\left[u(i \mid k), u(i+1 \mid k), \ldots, u\left(N_{p}-1 \mid k\right)\right]^{\top}$, where $\tilde{u}(k)$ is the decision sequence of $N_{p}$ predicted control inputs where $N_{p}$ is number of predication horizon. Moreover, $w_{1} \in \mathbb{R}^{n_{x} \times n_{x}}$ and $w_{2} \in \mathbb{R}^{n_{u} \times n_{u}}$ are positive definite weighting matrices that establish the trade-off between state and the control input effort, respectively. The super-index $p$ is the squared norm that is used for this paper. Furthermore, the sets $\mathbb{X}$ and $\mathbb{U}$ are defined as

$$
\begin{aligned}
& x \in \mathbb{X} \triangleq\left\{x(k) \in \mathbb{R}^{n_{x}} \mid \underline{x} \leq x(k) \leq \bar{x}\right\}, \quad \forall k, \\
& u \in \mathbb{U} \triangleq\left\{u(k) \in \mathbb{R}^{n_{u}} \mid \underline{u} \leq u(k) \leq \bar{u}\right\}, \quad \forall k,
\end{aligned}
$$

where vectors $\underline{x} \in \mathbb{R}^{n_{x}}$ and $\bar{x} \in \mathbb{R}^{n_{x}}$ determine the minimum and maximum possible state values of the system, respectively. Similarly, $\underline{u} \in \mathbb{R}^{n_{u}}$ and $\bar{u} \in \mathbb{R}^{n_{u}}$ determine the minimum and maximum possible value of manipulated variables, respectively. The control law is applied in a receding horizon manner, that is, at time $k$ the optimal sequence of control input is defined and then, only the first optimal control input is applied to the system. Then, the new measurements are applied to update the initial conditions and then the optimization problem is solved again using the receding horizon rule. Also, $x(i \mid k)$ is the predicted state at time $i$, with $i=1, \ldots, N_{p}$, obtained by starting from the state $x(0 \mid k)=x(k)$.

The LPV model can not be evaluated before solving the optimization problem (2) because the future state sequence is not known. Indeed $x(i \mid k)$ depends not only on the future control inputs $u(k)$, but also on the future scheduling parameters $\theta(k)$ and delay, where for a general LPV system are not assumed to be known a priori but only to be measurable online at current time $k$. In addition, in the case of a system with varying delay, the delay varies with the scheduling variables. Hence, predicting the future states regarding the dynamic of the system is more difficult. But, for a quasi-LPV system, where the scheduling parameters $\theta(k)$ are defined by $x(k)$ and $u(k)$, the delay and state trajectory can be predicted.

\section{PROPOSED APPROACH}

This section proposes an MPC controller design in order to solve the optimization problem of an LPV model with parameter-varying delay where the parameters and delays change along the prediction horizon. The idea is to find a solution to the problem (2) by solving an online optimization problem as a QP problem. The solution for this problem is based on the estimation the scheduling variables and subsequently delay into the prediction horizon and using them to update the system matrices of the model that used by the MPC controller. In fact, the sequence of the control input is used to modify the delay and system matrices of the model used along the prediction horizon. Therefore, the sequence of states and predicted parameters can be obtained from the control sequence $\tilde{u}(k)$ as

$$
\tilde{\mathbf{x}}(k)=\left[\begin{array}{lll}
x(i+1 \mid k) & x(i+2 \mid k) \cdots x\left(N_{p} \mid k\right)
\end{array}\right]^{T} \in \mathbb{R}^{N_{p} n_{x}},
$$

$\Theta(k)=\left[\begin{array}{lll}\hat{\theta}(i \mid k) & \hat{\theta}(i+1 \mid k) \cdots \hat{\theta}\left(N_{p}-1 \mid k\right)\end{array}\right]^{T} \in \mathbb{R}^{N_{p} n_{\theta}}$.

Since the delays depend on the scheduling parameters, the delays can be estimated based on the sequence of predicted parameters as

$$
\tilde{\tau}(k)=\left[\begin{array}{lll}
\tau(i \mid k) & \tau(i+1 \mid k) \cdots \tau\left(N_{p}-1 \mid k\right)
\end{array}\right]^{T} \in \mathbb{R}^{N_{p} n_{\tau}} .
$$

Thus, with slight abuse of notation, $\psi$ and $\phi$ can be used as: $\Theta(k)=\psi\left(\left[x^{\top}(k) \quad \tilde{\mathbf{x}}^{\top}(k)\right]^{\top}, \tilde{u}(k)\right)$ and $\tilde{\tau}(k)=$ $\phi\left(\left[\begin{array}{ll}x^{\top}(k) & \tilde{\mathbf{x}}^{\top}(k)\end{array}\right]^{\top}, \tilde{u}(k)\right)$, respectively. The vector $\Theta(k)$ includes parameters from time $k$ to $k+N_{p}-1$ whilst the state prediction is accomplished for time $k+1$ to $k+N_{p}$.

Consequently, by using the vectors (4) and (5), the $\tilde{\mathbf{x}}(k)$ can be simply formulated as follow

$$
\begin{aligned}
\tilde{\mathbf{x}}(k)= & \mathcal{A}(\Theta(k)) x(k)+\mathcal{A}_{\tau}(\Theta(k)) x(k-\tilde{\tau}(\Theta(k))) \\
& +\mathcal{B}(\Theta(k)) \tilde{u}(k)+\mathcal{B}_{\tau}(\Theta(k)) \tilde{u}(k-\tilde{\tau}(\Theta(k))),
\end{aligned}
$$

where $\mathcal{A}$ and $\mathcal{A}_{\tau} \in \mathbb{R}^{n_{x} \times n_{x}}$ and $\mathcal{B}$ and $\mathcal{B}_{\tau} \in \mathbb{R}^{n_{x} \times n_{u}}$ are given by (7) and (8). By using (6) and augmented block diagonal weighting matrices $\tilde{w}_{1}=\operatorname{diag}_{N_{p}}\left(w_{1}\right)$ and $\tilde{w}_{2}=$ $\operatorname{diag}_{N_{p}}\left(w_{2}\right)$, the cost function (2a) can be represented in vector form as

$$
\min _{\tilde{u}(k)} J(k)=\left(\tilde{\mathbf{x}}(k)^{\top} \tilde{w}_{1} \tilde{\mathbf{x}}(k)+\tilde{u}(k)^{\top} \tilde{w}_{2} \tilde{u}(k)\right),
$$

subject to

$$
\begin{array}{cl}
u(i \mid k) \in \mathbb{U}, & x(i \mid k) \in \mathbb{X}, \\
x(0 \mid k)=x(k) & \theta(0 \mid k)=\theta(i \mid k),
\end{array}
$$




$$
\mathcal{A}(\Theta(k))=\left[\begin{array}{c}
I \\
A(\hat{\theta}(k)) \\
A(\hat{\theta}(k+1)) A(\hat{\theta}(k)) \\
\vdots \\
A\left(\hat{\theta}\left(k+N_{p}-1\right)\right) A\left(\hat{\theta}\left(k+N_{p}-2\right)\right) \ldots A(\hat{\theta}(k))
\end{array}\right]
$$

and

$$
\mathcal{B}(\Theta(k))=\left[\begin{array}{ccccc}
0 & 0 & 0 & \ldots & 0 \\
B(\hat{\theta}(k)) & 0 & 0 & \ldots & 0 \\
A(\hat{\theta}(k+1)) B(\hat{\theta}(k)) & B(\hat{\theta}(k+1)) & 0 & \ldots & 0 \\
\vdots & \vdots & \ddots & \ddots & \vdots \\
A\left(\hat{\theta}_{k+N_{p}-1}\right) \ldots A(\hat{\theta}(k+1)) B(\hat{\theta}(k)) & A\left(\hat{\theta}_{k+N_{p}-1}\right) \ldots A(\hat{\theta}(k+2)) B(\hat{\theta}(k+1)) & \ldots & \left.B\left(\hat{\theta}_{k+N_{p}-1}\right)\right) & 0
\end{array}\right] .
$$

for all $i \in \mathbb{Z}_{\left[0, N_{p}-1\right]}$. Since the predicted states $\tilde{\mathbf{x}}(k)$ in (6) are linear in control inputs $\tilde{u}(k)$, the optimization problem can be solved as a QP problem, which is significantly easier than solving a nonlinear optimization problem.

However, the variation of delay at each iteration and inside the prediction horizon makes difficulty to solve the optimization problem considering a specific value of the prediction horizon length because for solving the optimization problem as MPC design based on a model with delay, the length of prediction horizon should include delay. Hence, during solving the MPC optimization problem the prediction horizon length should be adapted considering the delay value. Thus, because of the change of the prediction horizon, the size of states and inputs vectors should be adapted accordingly. In order to summarize the proposed method, Algorithm 1 is introduced.

\section{CAse Study Application}

\section{A. Case study description}

In order to show the effectiveness of the proposed approach in the real case study, the pasteurization process that is described in [12] is chosen as a real case study. The pasteurization model is represented in terms of behavioral equations of each subsystem, consisting of holding tube, power, water pump, heat exchanger and hot water tank. The non-liner model of the pasteurization system is considered as

$$
\dot{x}=f(x, x(t-\tau), u, u(t-\tau), \omega(t)),
$$

where, $x=\left[\begin{array}{lllll}T_{1} & T_{2} & T_{2 r} & T_{4} & T_{i n}\end{array}\right]^{\top} \in \mathbb{R}^{5}, u=\left[\begin{array}{lll}N_{1} & N_{2} P\end{array}\right]^{\top} \in$ $\mathbb{R}^{3}$ and $\omega=\left[T_{a}\right] \in \mathbb{R}^{1}$ are states, inputs and disturbance of the pasteurization system, respectively. In addition, state equation $f: \mathbb{X} \times \mathbb{U} \longrightarrow \mathbb{X}$ and $\mathbb{X} \subseteq \mathbb{R}^{5}$ is presented in Appendix. The physical properties, process data and parameters related of the pasteurization model in the identification process are described in Table II and III in Appendix.

The identification procedure to estimate some unknown parameters of the nonlinear model is based on [13]. The idea of the method is to compare, the data obtained from the real set-up with the data obtained by simulating part of the continuous time non-linear model of the pasteurization process. This is formulated as an optimization problem that allows finding the parameter values that better approximate the real system in the least squares sense. Due to the lake of space, this can be only illustrated briefly by estimating unknown parameters of the hot-water tank temperature. The identification procedure is based on the knowledge of the non-linear model of the hot-water tank temperature (13b). It is assumed to have at access $N_{T_{2}}$ sets of data $P^{j}(k), N_{1}^{j}(k)$ for the hot-water tank temperature where $j=1, \ldots, N_{T_{2}}$ and $k=1, \ldots, K_{j}$ where $K_{j}$ is the number of samples of the $j$-th set of data. The identification process determines the minimum of the following objective function over the unknown parameters $\alpha_{1}, \alpha_{2}$ and $\alpha_{3}$ :

$$
J_{T_{2}}=\sum_{j=1}^{N_{T_{2}}} \sum_{k=1}^{K_{j}}\left(T_{2}^{j}(k)-\hat{T}_{2}^{j}(k)\right)^{2},
$$

where $\hat{T}_{2}^{j}(k)$ is the simulation provided by (13b). In the same manner, the identification procedure is applied based on the other equation of the nonlinear model for estimating the remaining unknown parameters. By using the non-linear embedding approach [14], the state-space LPV model of the pasteurization plant can be expressed as follows:

$$
\begin{gathered}
A=\left[\begin{array}{ccccc}
a_{11} & 0 & 0 & 0 & 0 \\
0 & a_{22} & a_{23} & 0 & 0 \\
0 & a_{32} & a_{33} & a_{34} & a_{35} \\
0 & a_{42} & a_{43} & a_{44} & a_{45} \\
0 & 0 & a_{53} & 0 & a_{55}
\end{array}\right], B=\left[\begin{array}{ccc}
b_{11} & 0 & 0 \\
0 & b_{22} & b_{23} \\
b_{31} & b_{32} & 0 \\
b_{41} & b_{42} & 0 \\
b_{51} & 0 & 0
\end{array}\right] \\
A_{\tau}=\left[\begin{array}{ccccc}
0 & a_{\tau, 12} & a_{\tau, 13} & a_{\tau, 14} & a_{\tau, 15} \\
0 & 0 & 0 & 0 & 0 \\
0 & 0 & 0 & 0 & 0 \\
0 & 0 & 0 & 0 & 0 \\
0 & 0 & 0 & 0 & 0
\end{array}\right], B_{d}=\left[\begin{array}{c}
b_{d, 11} \\
b_{d, 21} \\
b_{d, 31} \\
b_{d, 41} \\
0
\end{array}\right], \\
B_{\tau}=\left[\begin{array}{ccc}
b_{\tau, 11} & b_{\tau, 12} & 0 \\
0 & 0 & 0 \\
0 & 0 & 0 \\
0 & 0 & 0 \\
0 & 0 & 0
\end{array}\right], C=\left[\begin{array}{lllll}
1 & 0 & 0 & 0 & 0 \\
0 & 1 & 0 & 0 & 0
\end{array}\right],
\end{gathered}
$$

where the value of matrix parameters are introduced in Appendix. The most important objective of the pasteurization procedure is to ensure that the pasteurization temperature is attained and preserved as close the set-point amount for a 


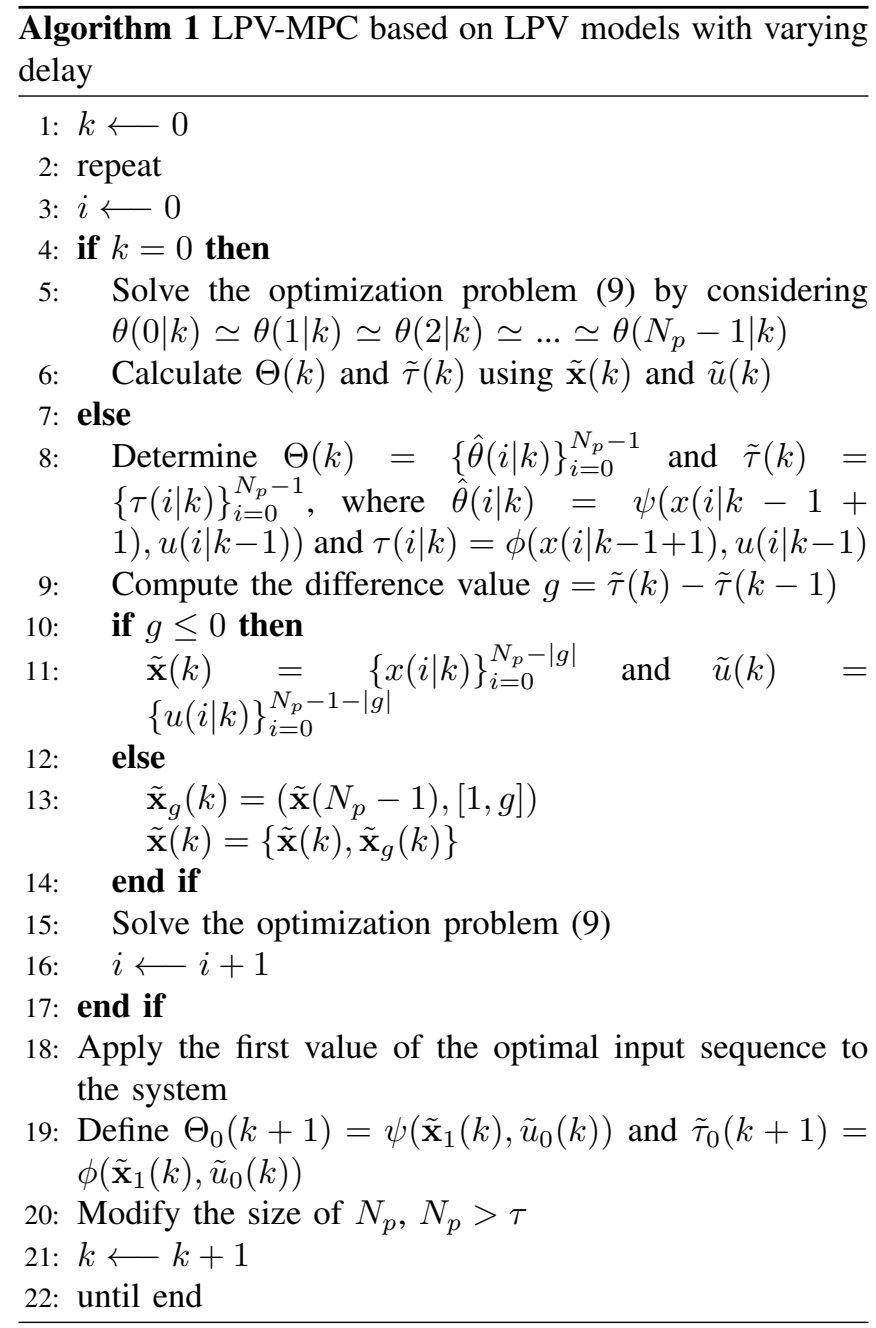

pre-established time. According to this, the $T_{1}$ temperature is the output of the holding tube for monitoring the temperature of the product after the pasteurization procedure, controlling $T_{1}$ to track set-points is one of the main objectives. On the other hand, the hot-water tank is the thermal energy source used to heat the product and proper system operation, $T_{2}$ should always be greater than $T_{1}$ due to achieve the final temperature desired. Hence, in order to guarantee the energy for the process, $T_{2}$ should be controlled.

\section{B. Results and Discussion}

In this section, the proposed algorithm based on the system with constant delay and varying delay is compared by the state-of-the-art NMPC approach based on the system with the same information. The comparison is made both in terms of closed-loop performance and computational timing performance. All simulation and computations have been carried out using a commercial computer with i7 $2.40-\mathrm{GHz}$ Intel core processor with 12 GB of RAM running MATLAB R2016b. The optimization problems (9) and (2) are solved by using the linear and nonlinear programming solvers available in YALMIP (Lofberg, 2004). The solution of (11) is found by means of fmincon MATLAB function of the Optimization
Toolbox. All tests are applied by same prediction horizon, parameters and constraints as mentioned in Table I.

In order to validate the values obtained by identification, the simulation of the non-linear model has been compared by the data obtained from the real system. Figures 1a) and $1 \mathrm{~b}$ ) present the comparison of the tracking response results that obtained under the LPV-based MPC approach and the NMPC controller based on the pasteurization model with the constant and varying delay during the prediction horizon, respectively. Moreover, the responses of the control actions using a controller based on proposed approach and NMPC controller that considered the model with the constant and varying delay in the prediction horizon are provided in Figures 1c) and 2, respectively. The comparison of computational timing performance of LPV-based MPC and NMPC is summarized in Table III. Actually, in the pasteurization process, the delay of the system is depended on the control action. Therefore, in the constant delay case, the delay of the system is varied at each time instant $k$, but during the prediction horizon are considered as constant delays, where the value of delay in the next iteration $k+1$ is computed by the first elements of the optimal input. For more clarity of how the delay is varied in the new proposed approach during prediction horizon, Figure 3 shows the evolution of delay when it is considered constant and varying during the prediction horizon.

According to these results, it can be observed that the proposed LPV-based MPC controller is tracked and reached the set-point and the performance of the proposed algorithm is almost the same as the NMPC one. In terms of the computational timing performance, there is a quite clear difference: although the average time proposes that the LPVbased MPC is on average four times faster than the NMPC, in fact, the algorithms are only as good as its worst-case performance, in which case it is clear that the proposed approach is approximately an order of magnitude faster. To sum up, the simulation results show that the proposed LPVbased MPC controller is able to control LPV time-delay systems while improving the performance of the closed-loop system and achieving the specified set-point.

\section{CONCLUSIONS}

This paper focused on the design of a model predictive control algorithm based on a class of Linear Parameter Varying (LPV) models with parameter varying delay. The constrained optimization problem for an LPV model with parameter varying and constant delay is solved iteratively by a series of QP problems while the scheduling parameters and delay are calculated at each time instant. The model with varying delay is predicted in the horizon by using the previous sequence of scheduling variable and state of the model. Based on the variation of the delay, the prediction horizon length is changed during the simulation. The proposed approach is considered a fast computation time and appreciably faster than other mentioned approach. For future research, It would be interesting to implement the proposed approach on the real benchmark of the pasteurization plant 


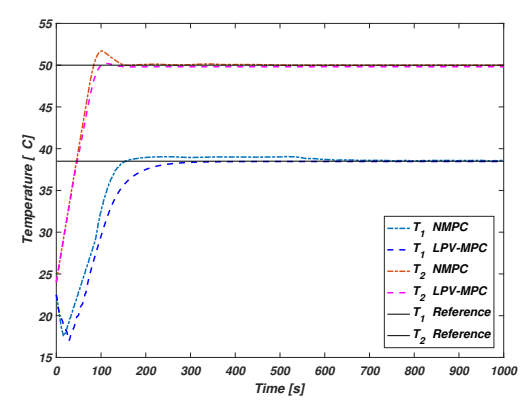

(a)

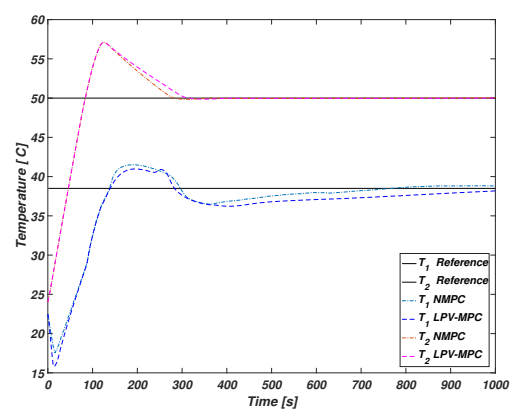

(b)

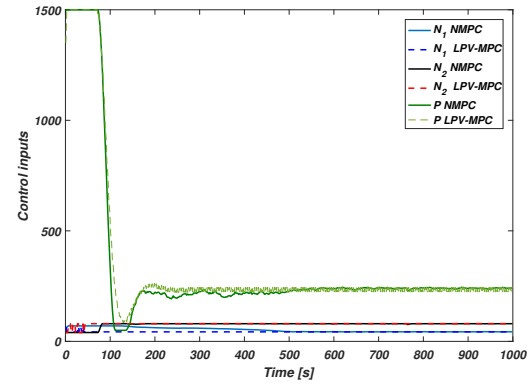

(c)

Fig. 1: Different assessments. (a) output temperatures (Delay is constant during the prediction horizon). (b) control inputs (Delay is varied during the prediction horizon). (c) control inputs (Delay is constant during the prediction horizon).

TABLE I: Comparison of each strategies timing performance

\begin{tabular}{lccccc}
\hline Configuration & Maximum time & Average time & Standard deviation & r.m.s. error $\left(T_{1}\right)$ & r.m.s. error $\left(T_{2}\right)$ \\
\hline NMPC constant delay into $N_{P}$ & 4.4540 & 2.0091 & 0.9071 & 4.9703 & 4.3479 \\
LPV-MPC constant delay into $N_{P}$ & 0.1640 & 0.0785 & 0.0078 & 5.243 & 4.425 \\
NMPC varying delay into $N_{P}$ & 24.887 & 6.9558 & 3.9441 & 5.117 & 4.7791 \\
LPV-MPC varying delay into $N_{P}$ & 0.8771 & 0.2480 & 0.1409 & 5.402 & 4.820 \\
\hline
\end{tabular}

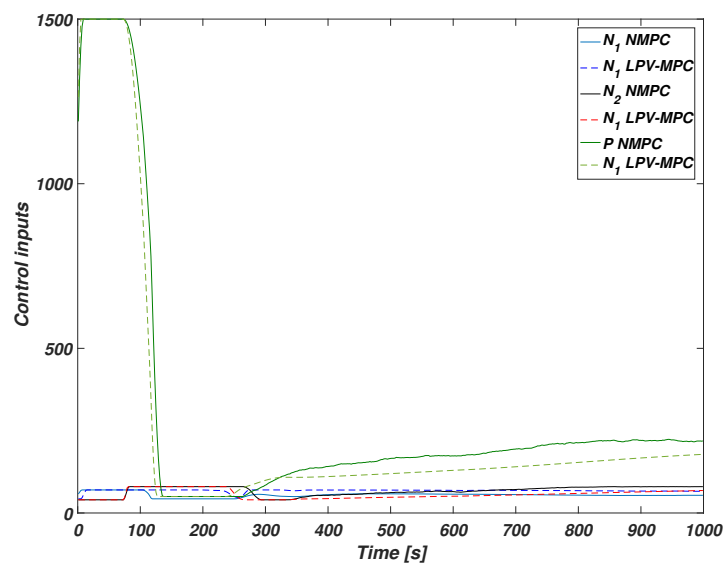

Fig. 2: Evaluation of the control inputs (Delay is varied during the prediction horizon)

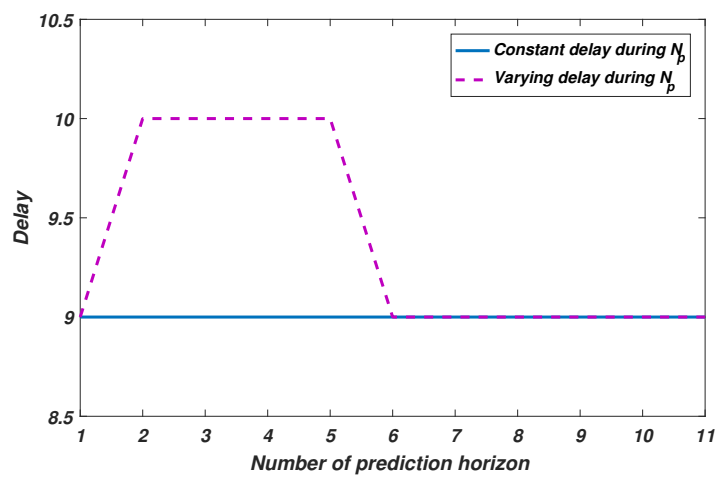

Fig. 3: Comparison of delay during the prediction horizon by considering the uncertainty and sensitivity of disturbance in the model.

\section{APPENDIX}

For obtaining the pasteurization model, physical principle based fundamental laws such as energy balances and heat exchanger design are utilized to describe the main processes of the plant. Therefore, Energy balance and Bernoulli's law produces the following set of dynamical equations:

$$
\begin{aligned}
\frac{d T_{1}}{d t}= & \frac{2}{M_{1} C_{P}}\left(-U A \frac{T_{1}(t)+T_{4}(t-\tau)}{2}-T_{a}\right) \\
& -F_{1} C_{p}\left(T_{1}(t)-T_{4}(t-\tau)\right)-\frac{d T_{4}(t-\tau)}{d t} \\
\frac{d T_{2}}{d t}= & \alpha_{1} P(t)-F_{2} C_{p}\left(T_{2}(t)-T_{2} r(t)\right)-\alpha_{2} T_{2}(t)+\alpha_{3}
\end{aligned}
$$

$$
\begin{aligned}
\frac{d T_{2 r}}{d t}= & F_{1}\left(T_{4}(t)-T_{i n}(t)\right)+\alpha_{4} F_{2}\left(T_{2}(t)-T_{2 r}(t)\right) \\
& -\alpha_{5}\left(T_{2}(t)-T_{a}\right)-\alpha_{6}-\alpha_{7} N_{1}(t) \\
& \left(T_{4}(t)-T_{i n}(t)\right)+\alpha_{8} N_{2}(t)\left(T 2(t)-T_{2 r}(t)\right) \\
& -\alpha_{9}\left(T_{4}(t)-T_{i n}(t)\right)+\alpha_{10}\left(T_{2}(t)-T_{2 r}(t)\right)
\end{aligned}
$$

$$
\begin{aligned}
\frac{d T_{4}}{d t}= & -F 1\left(T_{4}(t)-\operatorname{Tin}(t)\right)+\alpha_{11} F_{2}\left(T_{2}(t)-T 2 r(t)\right) \\
& -\alpha_{12}-\alpha_{13} N_{1}(t)\left(T_{4}(t)-\operatorname{Tin}(t)\right) \\
& +\alpha_{14} N_{2}(t)\left(T_{2}(t)-T 2 r(t)\right)
\end{aligned}
$$

$$
\frac{d T_{i n}}{d t}=\frac{\alpha_{15}\left(N_{1}(t)-\alpha_{16}\right)\left(T_{1}(t)-T_{i n}(t)\right)}{M_{2}}
$$


where $F_{1}=\alpha_{17}\left(N_{1}(t)-\alpha_{16}\right), F_{2}=\alpha_{18}\left(N_{2}(t)-\alpha_{19}\right)$, and the delay of system is

$$
\begin{aligned}
\tau(t)= & -\alpha_{20}-\alpha_{21}\left(N_{1}(t)\right)^{3}+\alpha_{22}\left(N_{1}(t)\right)^{2} \\
& -\alpha_{23} N_{1}(t)+\alpha_{24} .
\end{aligned}
$$

The value of entries for the state-space matrices for the controller used in (12) are presented as follows:

$$
\begin{aligned}
a_{11} & =\frac{-U A}{M_{1} C_{p}}-F_{1} C_{p} \\
a_{22} & =-F_{2} C_{p}-\alpha_{2} \\
a_{23} & =F_{2} C_{p} \\
a_{32} & =F_{2} \alpha_{4}+N_{2}(k) \alpha_{8}+\alpha_{10}-\alpha_{5} \\
a_{33} & =-F_{2} \alpha_{4}-N_{2}(k) \alpha_{8}-\alpha_{10} \\
a_{34} & =-N_{1}(k) \alpha_{7}+F_{1}-\alpha_{9} \\
a_{35} & =N_{1}(k) \alpha_{7}-F_{1}+\alpha_{9} \\
a_{42} & =F_{2} \alpha_{11}+N_{2}(k) \alpha_{14} \\
a_{43} & =-F_{2} \alpha_{11}-N_{2}(k) \alpha_{14} \\
a_{44} & =-N_{1}(k) \alpha_{13}-F_{1} \\
a_{45} & =N_{1}(k) \alpha_{13}+F_{1} \\
a_{53} & =\frac{\alpha_{15}\left(N_{1}(k)-\alpha_{16}\right)}{M_{2}} \\
a_{55} & =\frac{-\alpha_{15}\left(N_{1}(k)-\alpha_{16}\right)}{M_{2}} \\
b_{11} & =-\alpha_{17} C_{p}\left(T_{1}(k)-T_{4}(k-\tau)\right) \\
b_{22} & =-\alpha_{18} C_{p}\left(T_{2}(k)-T_{2 r}(k)\right) \\
b_{23} & =\alpha_{1} \\
b_{31} & =\left(\alpha_{17}-\alpha_{7}\right)\left(T_{4}(k)-T_{i n}(k)\right) \\
b_{32} & =\left(\alpha_{18} \alpha_{14}+\alpha_{8}\right)\left(T_{2}(k)-T_{2 r}(k)\right) \\
b_{41} & =\left(-\alpha_{17}-\alpha_{7}\right)\left(T_{4}(k)-T_{i n}(k)\right) \\
b_{42} & =\left(\alpha_{18} \alpha_{11}+\alpha_{14}\right)\left(T_{2}(k)-T_{2 r}(k)\right) \\
b_{51} & =\frac{\alpha_{15}\left(T_{2 r}(k)-T_{i n}(k)\right)}{M_{2}} \\
b_{d, 11} & =\alpha_{25} T_{a} \\
b_{d, 21} & =\alpha_{3} \\
b_{d, 31} & =\alpha_{5} T_{a}-\alpha_{6} \\
b_{d, 41} & =\alpha_{12} \\
a_{\tau, 12} & =-F_{2} \alpha_{11}-N_{2}(k) \alpha_{14} \\
a_{\tau, 13} & =F_{2} \alpha_{11}+N_{2}(k) \alpha_{14} \\
a_{\tau, 14} & =\frac{U A}{M_{1} C_{p}}+F_{1} C_{p}+F_{1}+\alpha_{13} N_{1}(k) \\
a_{\tau, 15} & =\alpha_{13} N_{1}(k)-F_{1} \\
b_{\tau, 11} & =\left(\alpha_{17}+\alpha_{13}\right)\left(T_{4}(k-\tau)-T_{i n}(k-\tau)\right) \\
b_{\tau, 12} & =\left(-\alpha_{11} \alpha_{18}-\alpha_{14}\right)\left(T_{2}(k-\tau)-T_{2 r}(k-\tau)\right) \\
a_{1} & =a_{1}(k)
\end{aligned}
$$

Moreover, Tables II and III collect the information about both the model and simulation parameters, respectively.

TABLE II: Physical properties and process data

\begin{tabular}{llcc}
\hline Parameter & Description & Value & Unit \\
\hline$U$ & constant of convective heat transfer & 10 & {$\left[\mathrm{Wm}^{2} / \mathrm{K}\right]$} \\
$A$ & area of the tank & 0.0248 & {$\left[\mathrm{~m}^{2}\right]$} \\
$C_{p}$ & specific heat of the hot-water & 4.186 & {$\left[\mathrm{~J} / \mathrm{g}{ }^{\circ} \mathrm{C}\right]$} \\
$M_{1}$ & mass of liquid inside the tank & 82 & {$[\mathrm{~g}]$} \\
$M_{2}$ & mass product inside the regeneration section & 24.85 & {$[\mathrm{~g}]$} \\
$T_{a}$ & room temperature & 24.5 & {$\left[{ }^{\circ} \mathrm{C}\right]$} \\
$T_{1}$ & temperature at the end of holding tube & {$[0,80]$} & {$\left[{ }^{\circ} \mathrm{C}\right]$} \\
$T_{2}$ & temperature inside hot water tank & {$[0,80]$} & {$\left[{ }^{\circ} \mathrm{C}\right]$} \\
$T_{2 r}$ & returned water temperature from the heat exchanger & {$[0,80]$} & {$\left[{ }^{\circ} \mathrm{C}\right]$} \\
$T_{4}$ & temperature at the exit of heat exchanger & {$[0,80]$} & {$\left[{ }^{\circ} \mathrm{C}\right]$} \\
$T_{i n}$ & temperature after regeneration section & {$[0,80]$} & {$\left[{ }^{\circ} \mathrm{C}\right]$} \\
$N_{1}$ & percentage speed of feeding pump & {$[40-80]$} & {$[\%]$} \\
$N_{2}$ & percentage speed of hot-water pump & {$[20-80]$} & {$[\%]$} \\
$P$ & power of the electric resistor & {$[0-1500]$} & {$[\mathrm{W}]$} \\
\hline
\end{tabular}

TABLE III: The adjusted parameters

\begin{tabular}{lccc}
\hline Parameter & Value & Parameter & Value \\
\hline$\alpha_{1}$ & $2.2 \times 10^{-4}$ & $\alpha_{14}$ & $4 \times 10^{-4}$ \\
$\alpha_{2}$ & $1.6 \times 10^{-4}$ & $\alpha_{15}$ & $3.96 \times 10^{-1}$ \\
$\alpha_{3}$ & $3.74 \times 10^{-3}$ & $\alpha_{16}$ & 16.8154 \\
$\alpha_{4}$ & $10^{-4}$ & $\alpha_{17}$ & $6.814 \times 10^{-2}$ \\
$\alpha_{5}$ & $3.09 \times 10^{-2}$ & $\alpha_{18}$ & $2.68 \times 10^{-1}$ \\
$\alpha_{6}$ & $1.44 \times 10^{-1}$ & $\alpha_{19}$ & 16.3567 \\
$\alpha_{7}$ & $1.8 \times 10^{-3}$ & $\alpha_{20}$ & $9.62 \times 10^{-4}$ \\
$\alpha_{8}$ & $4.8 \times 10^{-4}$ & $\alpha_{21}$ & 4 \\
$\alpha_{9}$ & $6.54 \times 10^{-2}$ & $\alpha_{22}$ & $1.87 \times 10^{-1}$ \\
$\alpha_{10}$ & $5.18 \times 10^{-3}$ & $\alpha_{23}$ & 11.81 \\
$\alpha_{11}$ & $2.5 \times 10^{-3}$ & $\alpha_{24}$ & 269.44 \\
$\alpha_{12}$ & $22 \times 10^{-2}$ & $\alpha_{25}$ & $5.8 \times 10^{-4}$ \\
$\alpha_{13}$ & $1.16 \times 10^{-3}$ & & \\
\hline
\end{tabular}

\section{REFERENCES}

[1] P. Segovia, L. Rajaoarisoa, F. Nejjari, E. Duviella, V. Puig, Inputdelay model predictive control of inland waterways considering the backwater effect, in: 2nd IEEE Conference on Control Technology and Applications, 2018, to appear.

[2] F. Karimi Pour, V. Puig, C. Ocampo-Martinez, Comparative assessment of lpv-based predictive control strategies for a pasteurization plant, in: Control, Decision and Information Technologies (CoDIT), 2017 4th International Conference on, IEEE, 2017, pp. 0821-0826.

[3] F. Karimi Pour, V. Puig, C. Ocampo-Martinez, Multi-layer healthaware economic predictive control of a pasteurization pilot plant, International Journal of Applied Mathematics and Computer Science 28 (1) (2018) 97-110.

[4] F. Karimi Pour, V. Puig, G. Cembrano, Health-aware lpv-mpc based on a reliability-based remaining useful life assessment, IFACPapersOnLine 51 (24) (2018) 1285-1291.

[5] Y. Ge, J. Wang, C. Li, Robust stability conditions for dmc controller with uncertain time delay, International Journal of Control, Automation and Systems 12 (2) (2014) 241-250.

[6] S. Lee, S. Jeong, D. Ji, S. Won, Robust model predictive control for lpv systems with delayed state using relaxation matrices, in: American Control Conference (ACC), 2011, IEEE, 2011, pp. 716-721.

[7] B. Capron, M. Uchiyama, D. Odloak, Linear matrix inequality-based robust model predictive control for time-delayed systems, IET control theory \& applications 6 (1) (2012) 37-50.

[8] S. C. Jeong, P. Park, Constrained mpc algorithm for uncertain timevarying systems with state-delay, IEEE Transactions on Automatic Control 50 (2) (2005) 257-263.

[9] B. Ding, L. Xie, W. Cai, Robust mpc for polytopic uncertain systems with time-varying delays, International Journal of Control 81 (8) (2008) 1239-1252.

[10] F. Wu, K. M. Grigoriadis, Lpv systems with parameter-varying time delays: analysis and control, Automatica 37 (2) (2001) 221-229.

[11] L. Zhang, W. Xie, Z. Zhong, J. Wang, Robust model predictive control synthesis for state-delayed systems with randomly occurring input saturation nonlinearities, Transactions of the Institute of Measurement and Control 40 (1) (2018) 179-190.

[12] F. Karimi Pour, C. Ocampo-Martinez, V. Puig, Output-feedback model predictive control of a pasteurization pilot plant based on an lpv model, in: Journal of Physics: Conference Series, Vol. 783, IOP Publishing, 2017, p. 012029.

[13] F. Nejjari, D. Rotondo, V. Puig, M. Innocenti, Quasi-lpv modelling and non-linear identification of a twin rotor system, in: Control \& Automation (MED), 2012 20th Mediterranean Conference on, IEEE, 2012, pp. 229-234.

[14] A. Kwiatkowski, M.-T. Boll, H. Werner, Automated generation and assessment of affine lpv models, in: Decision and Control, 2006 45th IEEE Conference on, IEEE, 2006, pp. 6690-6695. 